MINI-SYMPOSIUM

\title{
Stress myocardial contrast echocardiography
}

\section{J Monaghan}

$\mathrm{E}$ valuation of reversible ischaemia and understanding the physiological significance of known coronary lesions is one of the most important applications of functional cardiac testing. Myocardial perfusion abnormalities during stress are important predictors of clinical outcome and appear to be superior to the angiographic evaluation of the coronary anatomy alone. ${ }^{1-3}$

The classical ischaemic cascade illustrated in fig 1 demonstrates that one of the first indicators of an imbalance between myocardial oxygen demand and supply is a reduction in myocardial perfusion. Myocardial contrast echocardiography (MCE) has the ability to demonstrate both myocardial blood volume and velocity on a regional basis. The combination of these two parameters has been shown to represent myocardial blood flow $w^{4-7}$ and we can assume that this is directly proportional to myocardial perfusion. The excellent spatial resolution of MCE affords significant advantages over nuclear techniques. Cost, availability, and patient preference also means that stress MCE has the potential to be a more appropriate investigation than stress cardiac magnetic resonance imaging in the evaluation of reversible ischaemia.

\section{PRINCIPLES OF STRESS MCE}

Ischaemia, as generated during stress in myocardial segments subtended by epicardial coronary vessels with flow limiting lesions, results in a relative reduction in myocardial blood flow. ${ }^{8}$ Myocardial blood flow is directly proportional to the product of blood volume and velocity. ${ }^{9-12}$ Both these parameters can be readily evaluated and appreciated during stress MCE, using a variety of techniques. Myocardial contrast signal intensity is directly proportional to blood volume. Relative reduction in blood volume during ischaemia will cause a reduction in the contrast signal as illustrated in fig 2 .

MCE techniques can be described as either destructive or non-destructive imaging methods. ${ }^{4}$ The former techniques largely rely on contrast destruction to work and include

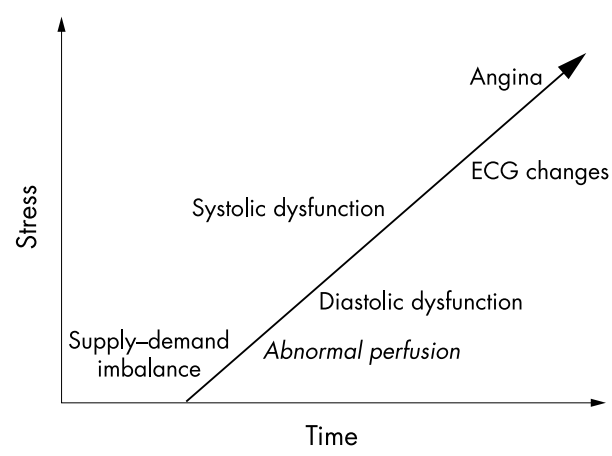

Figure 1 The classical ischaemic cascade demonstrating the sequence of events that occur during stress induced ischaemia. Abnormalities in myocardial perfusion occur earlier than mechanical or ECG changes. Therefore, methods which are able to detect stress induced perfusion abnormalities should have good sensitivity for diagnosing reversible ischaemia. harmonic imaging, harmonic power Doppler (angio), and pulse inversion imaging. These techniques operate at a high ultrasound transmit power (mechanical index (MI)) and need to utilise intermittent imaging. Therefore wall motion information is not available. Low output (low MI) techniques have also been developed. These methods minimise contrast destruction because of low power, thereby facilitating realtime imaging of myocardial contrast, together with wall motion and thickening information. Both of these types of techniques can be utilised for stress MCE studies.

Contrast agents can be administered either via a bolus injection or continuous infusion. Bolus injections have the advantages of using lower contrast volumes and are simple to administer. However, they often result in attenuation in the image plane for a transient period and there is often only a short period, during the decay phase, when the contrast agent concentration is appropriate for analysis of myocardial blood volume.

Infusions of contrast agents are more complex to administer and usually require a larger volume of agent. However, they require less operator involvement during the stress study, it is easier to adjust the infusion rate to optimise myocardial opacification without excessive attenuation, and

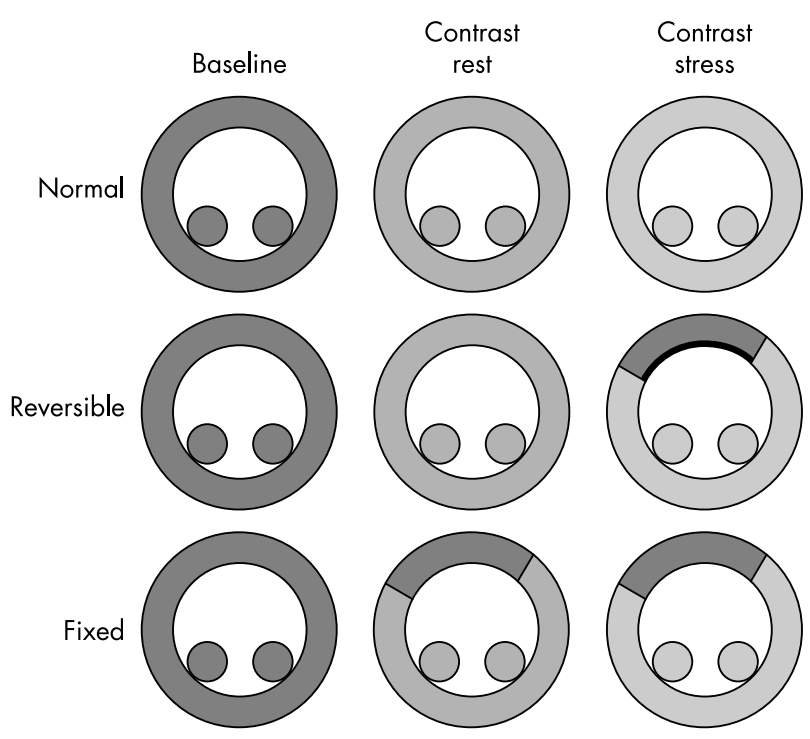

Figure 2 The basic principles of qualitative evaluation of stress MCE images. Following administration of contrast, the myocardial video intensity increases, representing contrast within the myocardial blood volume. During stress, myocardial blood volume should increase and therefore the myocardial signal intensity should be greater than at rest. In myocardial segments supplied by coronary vessels with reduced flow reserve (reversible ischaemia), myocardial blood volume will decrease, resulting in a reduction in the contrast signal. This is often most evident in the subendocardium where ischaemia is greatest. Myocardial segments with fixed perfusion defects will show a reduced contrast effect at both rest and stress when compared to normally perfused territories. 


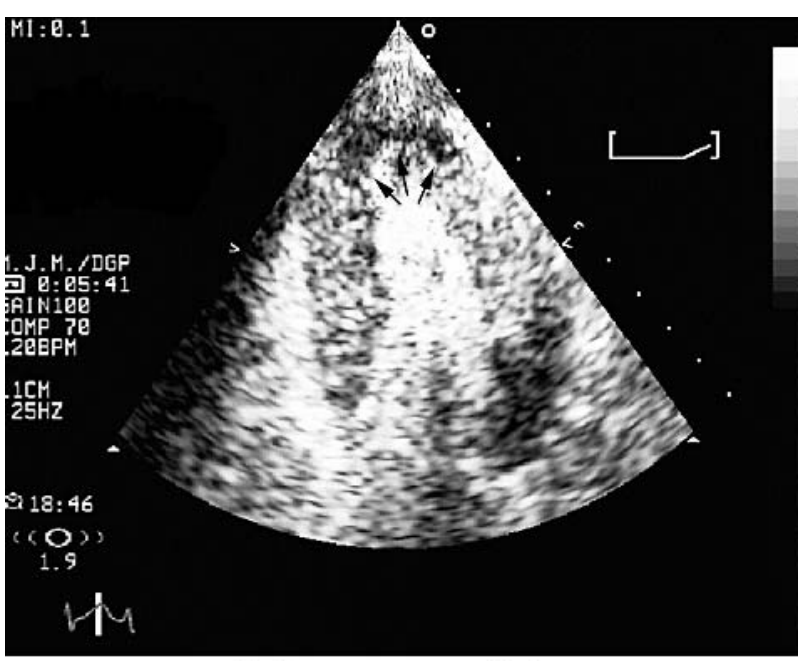

Peak stress + contrast Ap4c

Figure 3 End systolic apical four chamber image acquired during realtime low Ml imaging (power modulation) at peak dobutamine stress with a contrast bolus injection. This patient had had a left anterior coronary artery stent implanted eight weeks previously and had been referred for stress MCE because of a recurrence of his symptoms. An apical subendocardial perfusion defect (arrowed) is clearly seen. Reduced myocardial contrast in the basal lateral segments represents a contrast attenuation artefact.

finally, a constant contrast concentration is more suited to quantification of myocardial blood flow.

The final variable in the methodology for stress MCE is the choice of stress agent. The choice is really between a positive inotrope such as dobutamine or arbutamine, or a coronary vasodilator such as dipyridamole or adenosine. ${ }^{13-15}$

\section{BOLUS INJECTIONS WITH REAL-TIME IMAGING}

Bolus contrast injections are probably more suited to being used in combination with real-time, non-destructive, low MI imaging techniques. This has the advantage of allowing simultaneous evaluation of wall motion and perfusion, using contrast enhancement. These real-time low MI imaging techniques usually provide excellent endocardial definition at adequate frame rates. Myocardial perfusion information is therefore incremental to the wall motion data and this methodology provides an excellent starting point for performing stress MCE studies. ${ }^{16}$

During stress, we would expect changes in perfusion to be evident before wall motion abnormalities. ${ }^{6}$ Therefore, the stress MCE images can be taken, if required, at a submaximal stage.

This technique can be utilised successfully during conventional dobutamine stress echocardiography, since at low doses dobutamine acts as a coronary vasodilator, ${ }^{8}$ and during stress ischaemia changes in myocardial blood volume will occur, as described previously.

Figures 3 and 4 illustrate a case of a patient with an occluded left anterior descending (LAD) stent, where the distal part of the LAD is supplied by collaterals from the right coronary artery (fig 4B). The image shown is an apical four chamber view taken at peak dobutamine stress $(40 \mu \mathrm{g} / \mathrm{kg} /$ min) during a contrast bolus injection. Coronary flow reserve is clearly reduced in the distal LAD territory and the myocardial contrast signal is reduced in the apical subendocardial territory. A stress induced wall motion abnormality was also evident in the same region. The excellent spatial resolution afforded by MCE is illustrated in this case, where it is possible to see the reduction in contrast enhancement confined to the subendocardium.

\section{DESTRUCTION, REPERFUSION REAL-TIME IMAGING}

When low MI, real-time imaging is used during stress MCE it is best to employ a "negative bolus" technique in combination with a contrast infusion. Again, good endocardial definition is nearly always obtained. The low MI imaging methodology typically uses an output MI of $<0$.1. If a few frames of high output power $(\mathrm{MI}>1.0)$ are fired, then contrast microspheres will be destroyed. This usually results in the myocardial contrast effect disappearing, whereas the left ventricular cavity remains bright because the contrast concentration is significantly greater in the cavity. The washin curve of contrast into the myocardium following destruction can then be observed and measured. As previously described, the slope of the curve is proportional to blood flow velocity and the plateau to blood volume. The product of both parameters is proportional to absolute blood flow, which will be reduced in ischaemic zones. A comparison before and after stress (especially vasodilator) will permit an assessment of coronary blood flow reserve.

However, a semi-quantitative approach can also be taken by direct observation of the number of cardiac cycles taken for an individual segment to reperfuse. Delayed reperfusion (>3-4 cycles), especially in the subendocardium, is highly suggestive of ischaemia. During stress, increased myocardial blood flow should mean that an individual segment will reperfuse more quickly than at rest. Again this methodology can be used with both inotropic or vasodilator stress, as previously outlined.
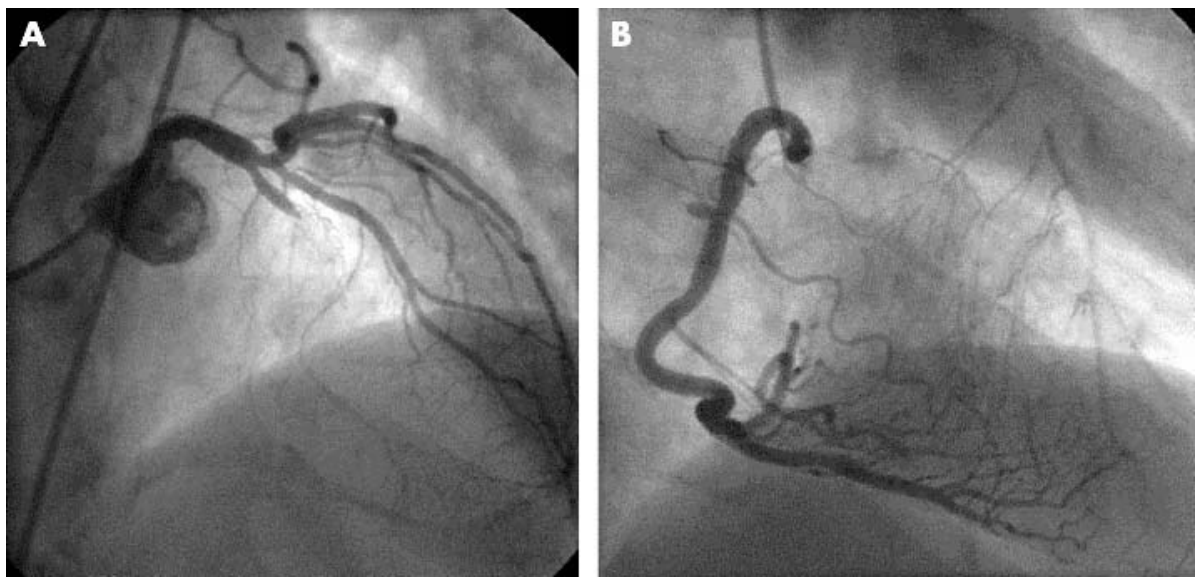

Figure 4 Coronary angiogram from the same patient shown in fig 3 . The left anterior descending artery is completely occluded at the location of the implanted stent. Right coronary angiography demonstrates collateral flow into the distal left anterior descending artery (beyond the stent). 


\section{DESTRUCTIVE, INTERMITTENT IMAGING}

As previously mentioned, this type of methodology will cause destruction of contrast microspheres within the myocardium and therefore these methods are utilised with intermittent imaging and no wall motion data are available.

By varying the triggering interval from one frame every cardiac cycle to up to one frame every 10 cycles it is possible to obtain data on myocardial blood flow. It is important to step though an acquisition sequence where the triggering interval is varied and increased in a stepwise fashion. Software on some ultrasound systems permits the creation of a triggering sequence which simplifies and standardises image acquisition.

Since myocardial blood flow increases during stress we would expect that reperfusion will occur with shorter trigger intervals than at baseline. For example, during vasodilator stress the normal coronary flow reserve response results in blood flow increasing by a factor of four. Therefore, at rest it may take a trigger interval of four cardiac cycles for an individual segment to reperfuse, whereas under maximum coronary vasodilation reperfusion should occur in one cycle.

Although this form of stress MCE is usually performed with vasodilator stress, it can also be utilised with inotropic stress. As previously mentioned, coronary physiology dictates that during stress induced ischaemia, relative reductions in myocardial blood volume, secondary to capillary de-recruitment, will result in slower reperfusion into ischaemic territories. Therefore, at short triggering intervals during stress, a perfusion defect may be visualised.

Although efforts to optimise stress MCE studies have so far mainly concentrated on evaluating perfusion in the left ventricular myocardium, it is possible to evaluate right ventricular myocardial perfusion. ${ }^{17}$ However, contrast attenuation is more problematic in the right ventricle and therefore careful attention to appropriate imaging planes are required.

\section{LIMITATIONS OF STRESS MCE}

Although excellent research data strongly suggests that these techniques will have a major clinical role in the future, current clinical practice has been slow to adopt stress MCE as an alternative method for evaluating reversible ischaemia. There are undoubtedly many reasons for this but some of the most important ones include the lack of available training in these techniques for non-invasive cardiologists, late development of appropriate contrast specific imaging software, lack of consensus about the appropriate combination of methodologies to perform a successful study, difficulties in obtaining reliable MCE data in the far zones of the ultrasound scan plane, and artefacts caused by contrast attenuation and wall motion, etc.

Despite these limitations, interest in this field remains extremely high and the remaining obstacles to progression of stress MCE are rapidly being overcome. The recent development of real-time three dimensional echo systems, that work effectively with contrast, creates the possibility of three dimensional stress MCE, which is a truly exciting prospect.

\section{CONCLUSION}

One of the earliest consequences of reversible myocardial ischaemia is a relative reduction in myocardial perfusion. MCE can detect myocardial contrast parameters which are directly related to perfusion at both rest and stress. This technology therefore has the potential to increase the diagnostic sensitivity for the detection of reversible ischaemia over and above that already established for conventional stress echocardiography. Since stress MCE can be used in combination with conventional stress echo techniques and can provide enhanced wall motion plus perfusion data, it should also increase our diagnostic confidence.

Finally, further important advantages over other competing technologies include cost, time, excellent spatial resolution, that it is a bedside technique, the ability to assess other intracardiac structures at the same time, and the lack of ionising radiation. For these reasons, stress MCE is likely to become the preferred technique for functional cardiac imaging in the future.

Correspondence to: Dr Mark J Monaghan, Cardiology Department, King's College Hospital, Denmark Hill, London SE5 9RS, UK; monaghan@compuserve.com

\section{REFERENCES}

1 Gibson RS, Watson DD, Craddock GB, et al. Prediction of cardiac events after uncomplicated myocardial infarction: a prospective study comparing predischarge exercise thallium 201 scintigraphy and coronary angiography. Circulation 1983;68:321-6.

2 Ladenheim ML, Kotler TS, Pollock BH, et al. Incremental prognostic power of clinical history, exercise electrocardiography and myocardial perfusion scintigraphy in suspected coronary artery disease. Am J Cardiol 1987;59:270-7.

3 Camarieri A, Picano E, Landi $P$, et al on behalf of investigators EPIC study: prognostic value of dipyridamole echocardiography early after myocardial infarction in elderly patients. J Am Coll Cardiol 1993;22:1809-15.

4 Becher H, Burns P. Handbook of contrast echocardiography. Berlin: Springer, 2000:62.

5 Wei K, Skyba DM, Firschke C, et al. Interactions between microbubbles and ultrasound: in-vitro and in-vivo observations. J Am Coll Cardiol 1997;29:1081-8.

6 Wei K, Jayaweera AR, Firoozan S, et al. Quantification of myocardial blood flow with ultrasound-induced destruction of microbubbles administered as a continuous venous infusion. Circulation 1998;97:473-83.

7 Wei K. Assessment of myocardial blood flow and volume using myocardial contrast echocardiography. Echocardiography 2002:19:409-16.

8 Leong-Poi H, Rim S, Le E, et al. Perfusion versus function: the ischaemic cascade in demand ischaemia. Circulation 2002;105:987-92

9 Masugata H, Peters B, Lafitte S, et al. Comparison of open- and closed-chest canine model for quantification of coronary stenosis severity by myocardial contrast echocardiography. Invest Radiol 2003;38:44-50.

10 Wei K, Jayaweera AR, Firoozan S, et al. Quantification of myocardial blood flow with ultrasound-induced destruction of microbubbles administered as a constant venous infusion. Circulation 1998:97:473-83.

11 Masugata H, Peters B, Lafitte S, et al. Quantitative assessment of myocardial perfusion during graded coronary stenosis by real-time myocardial contrast echo refilling curves. J Am Coll Cardiol 2001;37:262-9.

12 Galiuto L, May-Newman K, del Balzo U, et al. Assessment of coronary stenoses of graded severity by myocardial contrast echocardiography. J Am Soc Echocardiogr 2002;15:197-205.

13 Bin JP, Pelberg RA, Wei K, et al. Dobutamine versus dipyridamole for inducing reversible perfusion defects in chronic multivessel coronary artery stenosis. J Am Coll Cardiol 2002;40:167-74.

14 Morcerf F, Moraes A, Carrinho M, et al. Study of coronary flow reserve with intravenous use of microbubbles (contrast echocardiography) and adenosine: protocol for clinical application in patients suspected of having coronary heart disease. Arquivos Brasileiros de Cardiologia 2002;78:281-98.

15 Ronderos RE, Boskis M, Chung N, et al. Correlation between myocardial perfusion abnormalities detected with intermittent imaging using intravenous perfluorocarbon microbubbles and radioisotope imaging during high-dose dipyridamole stress echo. Clin Cardiol 2002;25:103-11.

16 Mor-Avi V, Caiani EG, Collins KA, et al. Combined assessment of myocardial perfusion and regional left ventricular function by analysis of contrastenhanced power modulation images. Circulation 2001;104:352-7.

17 Vargas-Barron J, Pena-Duque M, Roldan FJ, et al. Detection of right ventricular myocardial perfusion and contractile reserve by contrast echocardiography and low dose dobutamine in myocardial infarction after successful right coronary angioplasty. Archivos de Cardiologia de Mexico 2002;72:49-52. 